\title{
Performance of a solid-state frequency-shifted feedback laser in optical ranging
}

\author{
Cheikh Ndiaye \\ galas@ecei.tohoku.ac.jp \\ Takefumi Hara \\ tkfm@ecei.tohoku.ac.jp \\ Hiromasa Ito \\ h-ito@ecei.tohoku.ac.jp \\ Graduate School of Engineering, Tohoku University, JAPAN \\ Graduate School of Engineering, Tohoku University, JAPAN \\ Graduate School of Engineering, Tohoku University, JAPAN
}

The performance of a frequency-shifted feedback laser ( $\mathrm{FSFL}$ ) using a $\mathrm{Nd}: \mathrm{YVO}_{4}$ crystal as gain medium was investigated as light source in high accuracy optical ranging based on optical-frequency domain reflectometry (OFDR). The FSFL generates a comb of chirped frequency components over a bandwidth of $45 \mathrm{GHz}$ with chirp rates of $3.8 \times 10^{17} \mathrm{~Hz} / \mathrm{s}$. In OFDR, distance accuracies better than $25 \mu \mathrm{m}$ at a data measurement time of $2 \mathrm{~ms}$ were demonstrated at a standoff distance of $5 \mathrm{~m}$. The results show that the FSFL is a promising light source for high accuracy, high speed 3D measurement applications. [D0I: 10.2971/jeos.2009.09010]

Keywords: frequency-shifted feedback laser, solid-state laser, optical ranging, distance measurement

\section{INTRODUCTION}

A frequency-shifted feedback laser (FSFL) has a frequency shifter in its cavity such that the intracavity field is frequency shifted by a fixed amount at every round trip. As a consequence, this laser behaves differently from a conventional laser. In the continuous wave regime, an FSFL can generate a comb of chirped frequency components with very high chirp rates, over a broad bandwidth [1]-[4]. Such a light source is of great interest for high accuracy, high resolution ranging applications based on optical frequency-domain reflectometry (OFDR). These applications include remote sensing, fiber characteristics measurements in telecommunications, 3 dimensional (3D) shape measurements for quality inspection in industry, structural health monitoring.

The great potential of the FSFL as light source for these applications has motivated the study of numerous cavity configurations incorporating various gain media. Previously, FSFLs operating in the $1550 \mathrm{~nm}$ window using erbium-doped fibers [5] or semiconductor optical amplifiers [6] have been widely studied for their potential as light source in high accuracy optical ranging. Owing to their short cavity lengths, solid-state FSFLs operating at $1 \mu \mathrm{m}$ offer the possibility of generating a chirped comb with faster chirp rate than their fiber counterparts. Furthermore, by using these lasers as pump light source, FSFL operation in the green portion of the spectrum by second harmonic generation is of interest to achieve higher spatial resolution, higher distance accuracy. The spectral characteristics of the $\mathrm{Nd}: \mathrm{YVO}_{4}$ FSFL and its ability for long distance ranging has been extensively studied for ranging previously [7]; however, as with other FSFL, there remains some work to do on investigating the performance in long range, high accuracy ranging applications, namely 3D shape measurements.

\section{FREQUENCY-SHIFTED FEEDBACK LASER}

A schematic of the solid-state FSFL we used in these experiments is shown in Figure 1. The gain medium was a $1 \mathrm{~mm}$ $\mathrm{Nd}: \mathrm{YVO}_{4}$ crystal and was pumped by a fiber pigtailed laser diode (Lumics) generating an output of $3 \mathrm{~W}$ at $808 \mathrm{~nm}$. The $1064 \mathrm{~nm}$ beam was collimated using a $40 \mathrm{~mm}$ lens. The cavity was formed between a highly reflective mirror and an output coupler mirror having a reflectivity of $80 \%$. The frequency shifter was an acousto-optic modulator (AA Optoelectronics) generating a round trip frequency shift of $280 \mathrm{MHz}$. The modulator had a maximum diffraction efficiency of $80 \%$ with $1 \mathrm{~W}$ of RF input power. The cavity length was about $10 \mathrm{~cm}$.

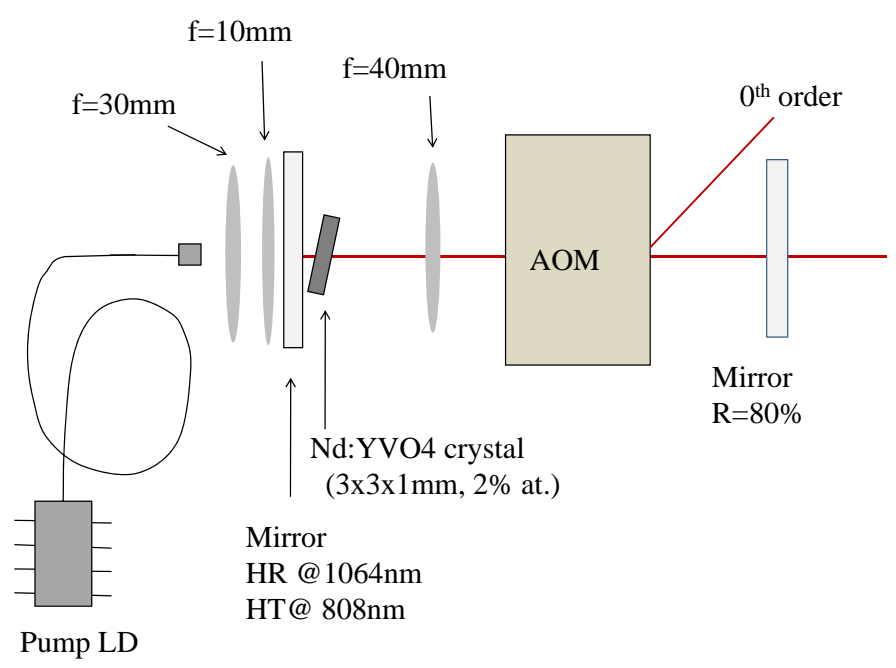

FIC. 1 Schematic of the $\mathrm{Nd:YVO} \mathrm{FSFL}_{4}$. 
Oscillation threshold of the FSFL was about $50 \mathrm{~mW}$, the slope efficiency was $15 \%$. Observations of the laser intensity using a digital oscilloscope showed that the FSFL tends to operate in the pulse regime at high pumping power (beyond $1 W)$. For the interferometric experiments described below, the laser was operated at moderate pumping powers, avoiding the pulse regime.

\section{PERFORMANCE OF THE FSFL IN RANGING}

\subsection{Optical spectral characteristics}

Figure 2 shows an optical spectrum of the FSFL centered at $1064.27 \mathrm{~nm}$. For high accuracy optical ranging using an FSFL, a broad bandwidth is mostly desired as indicated in the next section. The laser spectral profile was affected by many factors such as the gain profile, spectral filtering caused by some residual reflections from the output coupler or laser crystal facets. With adjustments of the pumping power or frequency shift, the FSFL optical spectrum was at times as broad as $60 \mathrm{GHz}$, however spectral distortions appear after some time, due to some instabilities. For more stable and accurate measurements, a stable and uniform spectrum is desirable. The laser parameters were adjusted to obtain a smooth spectrum with the broadest full width at half maximum (FWHM) of $0.18 \mathrm{~nm}(\sim 48 \mathrm{GHz})$. It is worth noting that these results show a significant improvement from earlier studies FSFL using $\mathrm{Nd}: \mathrm{YVO}_{4}$ in which the spectral widths were in the range of $10 \mathrm{GHz}$ [3]. We found that minimizing the residual reflections was of great importance in achieving broad bandwidths.

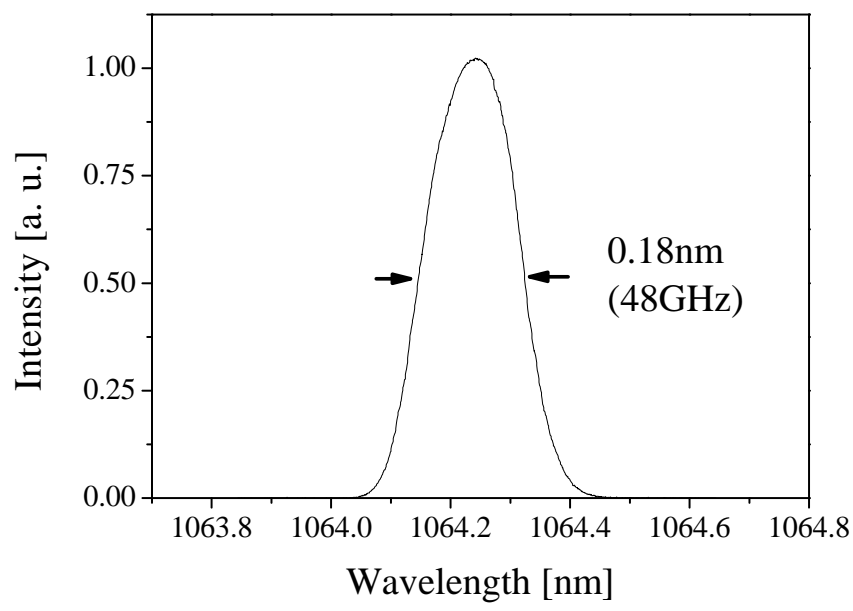

FIC. 2 Optical spectrum of the $\mathrm{Nd:YVO}{ }_{4}$ FSFL.

\subsection{OFDR with the FSFL}

A schematic of the OFDR setup using the FSFL as light source is shown in Figure 3. The FSFL output was collected with a collimator and split with a 1/99 fiber coupler. A small amount was taken as reference using a $3 \mathrm{~dB}$ coupler, while the bulk of the output $(\sim 40 \mathrm{~mW})$ was focused to a beam diameter of about $2 \mathrm{~mm}$ onto an aluminum plate placed at $5 \mathrm{~m}$, then the scattered light was sent to the $3 \mathrm{~dB}$ coupler via an optical circulator. All the fiber components were polarization maintained. The interferometer output was analyzed using either
Target

(Aluminum plate)

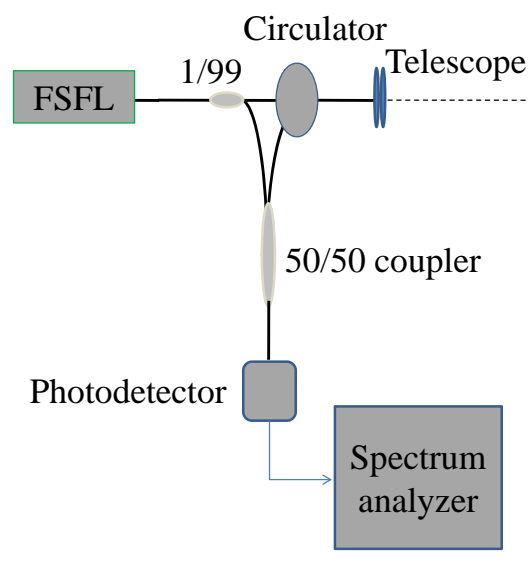

FIG. 3 Setup of the OFDR system.

a real-time or radio frequency (RF) spectrum analyzer after photodetection (Newfocus 1611).

Figure 4 shows the RF spectrum of the interferometer output observed with the RF spectral analyzer. It shows a beat signal at the cavity free-spectral range (FSR $=1.35 \mathrm{GHz}$ ), corresponding to a cavity length of $11 \mathrm{~cm}$. The beat signals $A$ and $\mathrm{B}$ were generated from interference between the delayed components of the chirped comb. The beats A were generated from the target, while those shown in B were from spurious reflections at the fiber end in the telescope. These interferometric beat signals can be expressed as [7]

$$
\begin{gathered}
v_{B m}=v_{s} v_{c} \frac{2 z}{c}-m v_{c} \\
m=v_{s} v_{c} \frac{\Delta v_{B m}}{\Delta v_{S}}-v_{c} v_{B m} .
\end{gathered}
$$

Here $v_{B m}$ is the beat frequency, $v_{S}$ is the frequency shift, $v_{c}$ is the FSR, $z$ is optical path length, $c$ is speed of light, $\Delta v_{B m}$ is the beat frequency variation when the frequency shift changes by $\Delta v_{s}, m$ is an integer representing the beat order. The distance is given by :

$$
z=\frac{c}{2 v_{s} v_{c}}\left(v_{B m}+m v_{c}\right)
$$

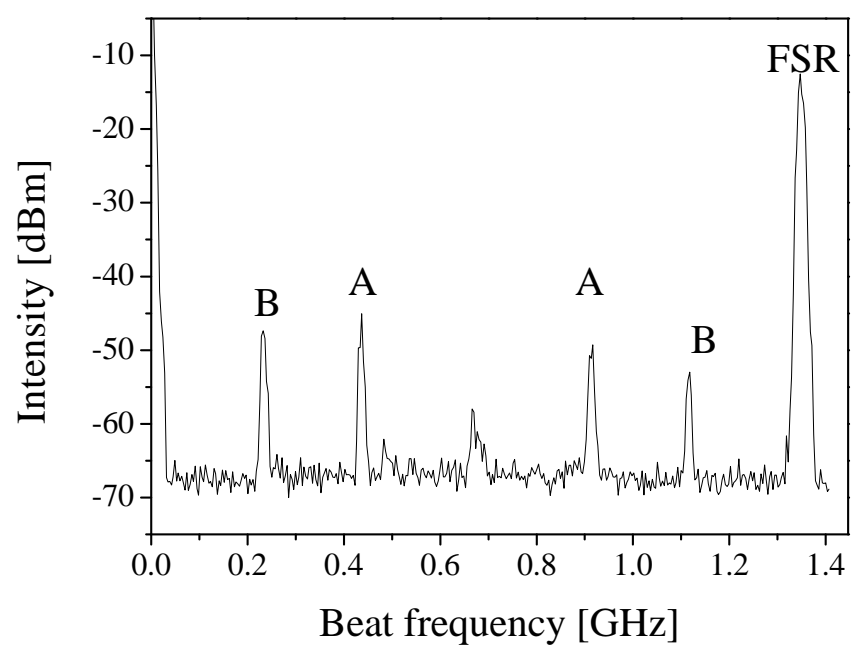

FIG. 4 RF spectrum of the interferometer output. Resolution was $1 \mathrm{MHz}$. 
Figure 5 shows the RF spectrum of one interferometric beat signal measured using the real-time spectrum analyzer. The resolution of the analyzer was $100 \mathrm{KHz}$ and the data were averaged 10 times. The measured beat bandwidth was approximately $8 \mathrm{MHz}$. If the FSFL spectral components chirp throughout the laser bandwidth $\Delta v$, at the rate $v_{s} v_{c}$, then after a Fourier transform at the spectrum analyzer, the beat bandwidth is approximately as $v_{s} v_{c} / \Delta v$. From the measured parameters, the calculated beat bandwidth is $7.8 \mathrm{MHz}$. Therefore we can state that the spectral components chirp continuously across the chirp range $\Delta v$, without phase discontinuity. The distance resolution $\delta \mathrm{z}=\mathrm{c} / 2 \Delta v$ is then approximately $3 \mathrm{~mm}$ in this case.

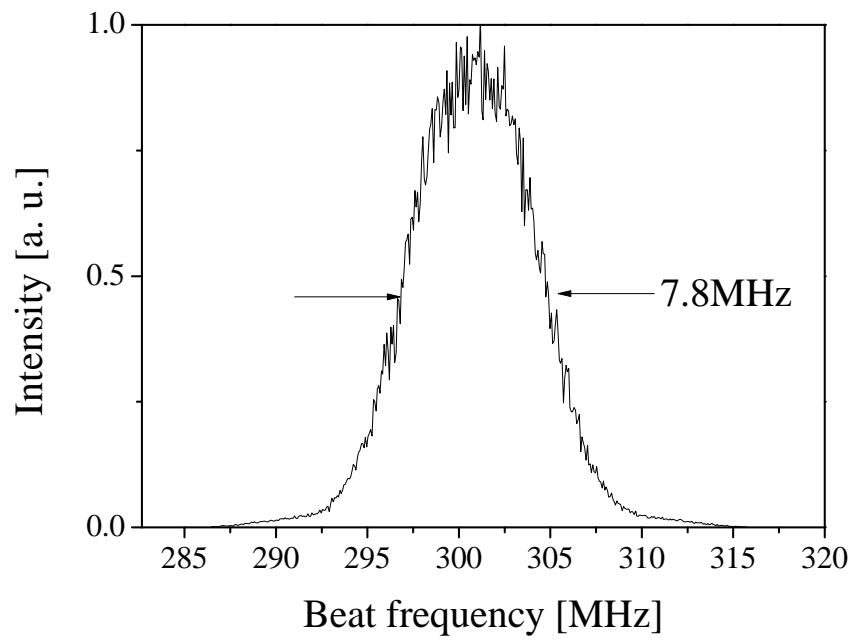

FIC. 5 RF spectrum of an interferometric beat signal after 10 averaging. Resolution was $100 \mathrm{KHz}$.

\subsection{Distance accuracy in FSFL ranging}

The distance accuracy is related to the uncertainty in determining the center frequency of the beat signal. Using Eq. (3), the uncertainty on the distance can be written as

$$
\begin{aligned}
\sigma^{2}(z)= & \frac{c^{2}}{4} \sigma\left(\frac{v_{B m}}{v_{c} v_{s}}+\frac{m}{v_{s}}\right)^{2} \\
\sigma^{2}(z)= & \frac{c^{2}}{4}\left\{\left(\frac{\sigma\left(v_{B m}\right)}{v_{c} v_{s}}\right)^{2}+\frac{v_{B m}^{2}}{v_{c}^{2} v_{s}^{2}}\left(\frac{\sigma^{2}\left(v_{c}\right)}{v_{c}^{2}}+\frac{\sigma^{2}\left(v_{s}\right)}{v_{s}^{2}}\right)\right. \\
& \left.+\left(\frac{\sigma(m)}{v_{s}}\right)^{2}+m^{2}\left(\frac{\sigma\left(v_{s}\right)}{v_{s}^{2}}\right)^{2}\right\}
\end{aligned}
$$

neglecting the uncertainty on $m$, this expression can be further simplified to

$$
\sigma^{2}(z)=\frac{c^{2}}{4}\left(\frac{\sigma\left(v_{B m}\right)}{v_{c} v_{s}}\right)^{2}+\frac{c^{2}}{4} \frac{v_{B m}^{2}}{v_{c}^{2} v_{s}^{2}}\left(\frac{\sigma^{2}\left(v_{c}\right)}{v_{c}^{2}}\right)+z^{2}\left(\frac{\sigma\left(v_{s}\right)}{v_{s}}\right)^{2}
$$

The uncertainty on the beat frequency $\sigma\left(v_{B m}\right)$ depends essentially on the beat bandwidth as well as the frequency resolution of the frequency measurement (spectrum analyzer in our case). This uncertainty can be expressed as [8]

$$
\sigma\left(v_{B m}\right) \approx \sqrt{\Gamma / T_{0}}
$$

where $\Gamma=v_{s} v_{c} / \Delta v$ is the beat bandwidth and $\mathrm{T}_{0}$ is the measurement time of the beat signal. Inserting this to Eq. (6) , a general expression for the uncertainty in the distance is

$$
\begin{aligned}
\sigma^{2}(z) \approx c^{2}\left(\frac{1}{4 v_{c} v_{s} \Delta v T_{0}}\right) & +c^{2} \frac{v_{B m}^{2}}{4 v_{c}^{2} v_{s}^{2}}\left(\frac{\sigma^{2}\left(v_{c}\right)}{v_{c}^{2}}\right) \\
& +z^{2}\left(\frac{\sigma\left(v_{s}\right)}{v_{s}}\right)^{2} .
\end{aligned}
$$

The term $\sigma\left(v_{s}\right) / v_{s}$ depends on the stability of the RF source used to drive the AOM. For stability levels of $10^{-7}$ or better, the distance does not significantly affect the accuracy for few tens of metres. Also by having the laser cavity stable enough, at distances of few meteres, the distance accuracy can be expressed as

$$
\sigma(z) \approx \frac{c}{2 \sqrt{v_{c} v_{s} \Delta v T_{0}}}
$$

To verify this result, we measured the distance accuracy at a standoff distance of $5 \mathrm{~m}$ in the laboratory environment. The peak frequency of the beat shown in Figure 5 was measured for 128 data points. The measurement time (time for Fourier Transform) for a single point was set at $2 \mathrm{~ms}$. Figure 6 is a plot of the distance accuracy as a function of data averaging time. For a measurement time of $2 \mathrm{~ms}$, the calculated accuracy using the laser parameter was $25 \mu \mathrm{m}$, which is in good agreement with the measured results. In 3D shape measurement in which we are more interested, this result corresponds to a distance acuracy of about $35 \mu \mathrm{m}$ for a measurement time of $1 \mathrm{~ms}$ or measurement speed of 1000 points/second. In these experiments the laps between data acquisition was long due to our spectrum analyzer and the program to acquire the data. Therefore at very long data averaging times, cavity instabilities become important and the distance accuracy is dominated by the second term of Eq. (8), reaching a limit at few micrometres. We believe that faster data acquisition and longer averaging could possibly lead to submicrometer accuracy at few seconds.

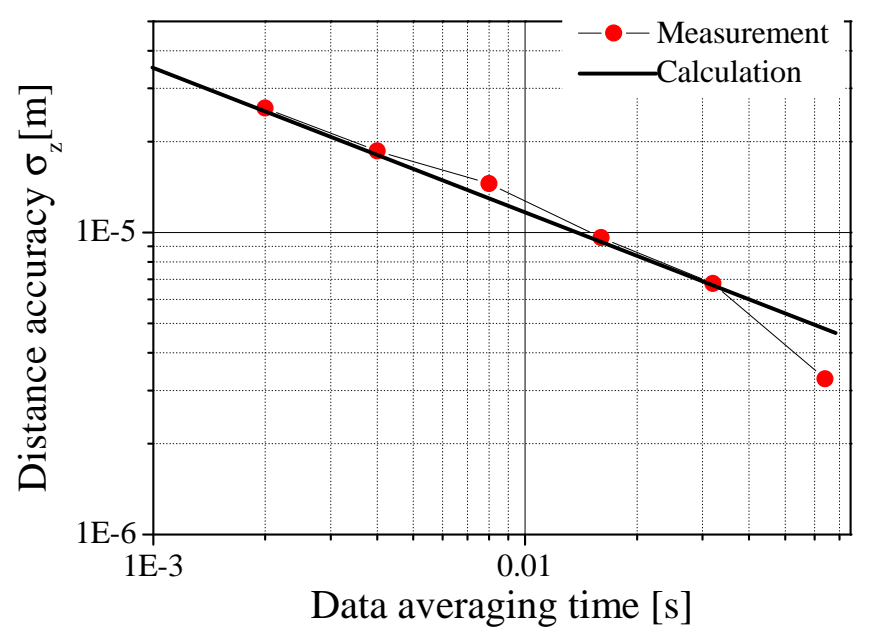

FIG. 6 Distance accuracy as a function of data averaging time.

As a demonstration of the system, small displacements were measured by moving the target by $50 \mu \mathrm{m}$ steps. 50 data points were measured with a single data point measurement time of $2 \mathrm{~ms}$. The relative distance variation is shown in Figure 7. By independently measuring the deviation from these data, the distance accuracy was $25 \mu \mathrm{m}$, which is in good agreement with the result previously obatined in Figure 6. 


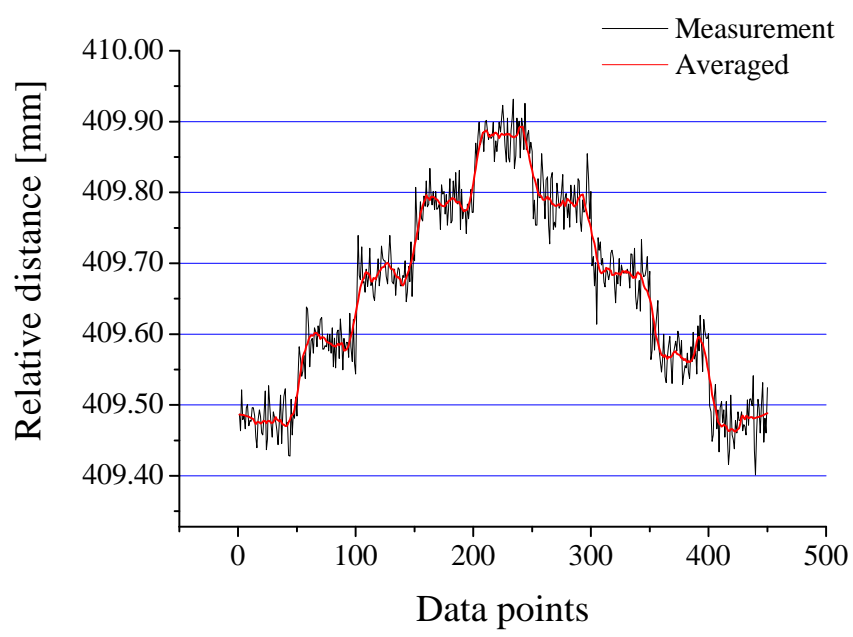

FIG. 7 Measurement of small displacements.

In summary a frequency-shifted feedback laser using a $\mathrm{Nd}: \mathrm{YVO}_{4}$ crystal as gain medium was investigated as light source in high accuracy optical ranging based on opticalfrequency domain reflectometry. Chirped comb generation over a bandwidth broader than $45 \mathrm{GHz}$ with chirp rates of $3.8 \times 10^{17} \mathrm{~Hz} / \mathrm{s}$ has been obtained. The performance of this laser in optical ranging was investigated and distance accuracies better than $25 \mu \mathrm{m}$ for measurement times of $2 \mathrm{~ms}$ were demonstrated, corresponding to measurement speeds of 500 points per second or $35 \mu \mathrm{m}$ at 1000 points $/ \mathrm{s}$. These results show the highpotentiality of the FSFL as light source for high accuracy and high speed 3D measurement applications.

\section{References}

[1] F. V. Kowalski, P. D. Hale, and S. J. Shattil, "Broadband ContinuousWave Laser" Opt. Lett. 13, 622-624 (1988).

[2] S. Balle, I. C. M. Littler, and K. Bergmann, "Frequency shifted feedback dye laser operating at a small shift frequency" Opt. Commun. 102, 166-174 ( 1993 ).

[3] K. Nakamura, F. Abe, K. Kasahara, T. Hara, M. Sato, and H. Ito, "Spectral Characteristics of an All Solid-State Frequency-Shifted Feedback Laser" IEEE J. Quantum Elect. 33, 103-111 (1997).

[4] F. V. Kowalski, C. Ndiaye, K. Nakamura, and H. Ito, "Noise waveforms generated by frequency shifted feedback lasers: application to multiple access communications" Opt. Commun. 231, 149-164 (2004)

[5] M. Yoshida, K. Nakamura, T. Miyahara, and H. Ito, CWE3 157 (European Conf. Lasers and Electro-Optics, Glasgow, 1998).

[6] C. Ndiaye, T. Hara, F. V. Kowalski, and H. Ito, "Spectral Characteristics of a Frequency-Shifted Feedback Ring Laser Using a Semiconductor Optical Amplifier" Jpn. J. Appl. Phys. 47, 3483-3485 (2008).

[7] K. Nakamura, T. Hara, M. Yoshida, T. Miyahara, and H. Ito, "Optical frequency domain ranging by a frequency-shifted feedback laser" IEEE J. Quantum Elect. 36, 305-316 (2000).

[8] L. P. Yatsenko, B. W. Shoreb, and K. Bergmann, "Ranging and interferometry with a frequency shifted feedback laser" 0pt. Commun. 242, 581-598 (2004). 